\title{
Chewable Gel Dosage Form
}

National Cancer Institute

\section{Source}

National Cancer Institute. Chewable Gel Dosage Form. NCI Thesaurus. Code C134876.

A formed or molded oral gel dosage form that maintains its shape, is elastic, and yields to mastication. 